\title{
International Committee for Standardization in Haematology: Protocol for type testing equipment and apparatus used for haematological analysis*
}

PREAMBLE This protocol is proposed as a procedure for type-testing (evaluation) equipment and apparatus used for clinical laboratory tests to ascertain the advantages and the limitations of such apparatus when used for specified tests under various circumstances. It is intended for use by reference laboratories appointed by international, national, or other appropriate authorities in the interest of the consumer. It includes analysis of results of measurements carried out by the manufacturers, which they are requested to provide to the appointed reference laboratory or laboratories. It is hoped that manufacturers will heed the requirement set out under 'Analysis of data and performance evaluation' when planning their own internal procedures of evaluation. When a particular type of instrument is shown by the reference laboratory to satisfy the performance criteria set out in the protocol this should be adequate to guarantee the reliability of that instrument in routine service.

This document is a consensus reached after discussions with individual colleagues and comment by national and international organisations representing the interests of the profession, health services, and manufacturers. The complete protocol may not necessarily be applicable for evaluation of the simpler types of instrument and, conversely, a more elaborate protocol may be required for evaluation of complex equipment systems, but all evaluations should follow the principles set out.

This tentative standard will be reviewed one year after publication, in accordance with the International Committee for Standardization in Haematology (ICSH) rules, before it is adopted as a definitive standard. Comment is invited. Correspondence should be addressed to $\mathrm{Dr} \mathrm{S}$. M. Lewis, ICSH Executive Secretary, Royal Postgraduate Medical School, London W12 0HS, United Kingdom.

\section{General information relating to instrument}

The following information should be given: name of instrument; manufacturer; representative in country; price (locally, as delivered); price of optional attachments; availability of rental or leasing arrangements; terms of guarantee; cost of maintenance contract, if available; cost of reagentsupply contract, if available; charges for service visits; length of time in use (excluding manufacturer's trials) in country of origin; approximate number in use and distribution; earlier models and/ or antecedent apparatus.

\section{Confirmation of specifications given by manufacturer}

\subsection{SPACE AND SERVICES REQUIREMENTS}

Space-Free standing or bench top, floor area, bench space, height, shelves, weight.

*ICSH Tentative Standard SC8-1/1977

Received for publication 30 August 1977
Services-Electricity: voltage, DC/AC, 50 or 60 cycles, amperage, limits of tolerated fluctuation; gas supply; water: pressure, quality; air and other gases (for example, $\mathrm{N}_{2}, \mathrm{CO}_{2}$ ): pressure, vacuum; drainage; gas exhaust.

Functional environment-Need for control of atmospheric environment; need for control of electrical interference.

Effects on environment-Level of disturbance (acoustical, vibration, heat generation); electrical and mechanical safety.

\subsection{INSTRUCTION BOOK}

Is it comprehensive, clearly written, in language of country? Does it include adequate information for servicing/fault finding? $\dagger$

†For an example of these requirements see NCCLS Standard ASI-1 1972, Preparation of manuals for installation, operation, and repair of laboratory instruments. Available from National Committee for Clinical Laboratory Standards, 771 E. Lancaster Avenue, Villanova, PA 19085, USA. 


\subsection{BASIC FACILITIES AND OPTIONAL EXTRAS}

\subsection{TRAINING}

What special training is required $(a)$ for routine use, (b) for maintenance?

Are training facilities provided locally by the manufacturer or his agent?

\subsection{INSTRUMENTATION}

Is the instrument intended for measuring one component or for multiple tests (concurrently or sequentially)?

Principle of (each) test performed.

Detailed specifications of instrument (for example, for colorimeter, if single or double beam, wavelength, band width, single or matched cuvettes, volume, flow-through system, light path length).

Degree of mechanisation and automation.

Input of samples of specimens.

\subsection{METHODS OF SPECIMEN IDENTIFICATION} Input facilities.

Output facilities.

\subsection{ANALYTICAL PROCEDURE}

\section{Co-ordination of channels.}

Can the programme selection be modified at the discretion of user?

Can one specimen be analysed preferentially if required?

Data presentation.

\subsection{RATE OF ANALYSIS}

Time between samples.

Throughput time.

Practical number of analyses per instrument/day.

Dces procedure require full-time or part-time technical attention?

Time for setting up and shutting down.

Effective working time/working cycle.

\subsection{SAMPLES}

Type of sample-Type of anticoagulant; concentration of anticoagulant; can prediluted sample of capillary blood be used?

Limitations of volume of sample-Facilities for rejection of unsuitable samples (haemolysed, clotted, etc.); handling of samples with extremes of analytic components and unusual constituents (for example, cold agglutinins, abnormal proteins).

2.10 REAGENTS, REFERENCE MATERIALS, AND CONTROLS

Reagents-What reagents are required? Storage requirements and shelf life of reagents. Are reagentso supplied only by manufacturer? Are suitable reagents available from other commercial suppliers Are formulae and preparation procedures made্ known by manufacturer? Can reagents be preparec들 by own laboratory from readily available chemicals and procedures (for example, filtration require $\frac{\mathbb{}}{\square}$ ments)? Volumes of reagents used for priming instrument and per run of 2,10 , and 50 specimens.

Reference materials-What reference materials ar provided by manufacturer? Are other materials available and suitable for use with instrument Method of instrument calibration.

Composition of tubing and other component parts oi machine-Will they stand common reagents, washinger and disinfection procedures?

2.11 ALARM SYSTEM FOR INSTRUMENT FAILUR OR DISTURBANCE

2.12 LIFE EXPECTANCY OF TUBING AND OTHE COMPONENTS

\section{Analysis of data and performance evaluation}

There are four aspects to this evaluation:

(1) Comprehensive statistical evaluation of pres cision, comparability, etc., by manufacturer.

(2) Assessment of manufacturer's data by refer ence laboratory after an independent statistical evaluation.

(3) Assessment of general performance of instrtio ment when used in a routine laboratory.

(4) Long-term follow-up of performance wites special reference to reliability and experience wit maintenance servicing after installation of a number of instruments in different laboratories.

3.1 EVALUATION BY MANUFACTURER For each item of equipment and for each analyticat process for which the instrument is intended the following data should be provided by the manuf facturer together with the relevant original testing protocol: (1) precision within a run, (2) precisio during a day, (3) precision from day to day,* (49) overall precision during the period of one month wit at least 2000 analyses, (5) estimate of short-tine drift, and (6) comparisons with a selected, wel震 known, and widely used ('established') method as described in a standard text book of haemato logical methods or with a reference method available. ICSH has already defined reference

*Specimens for these studies should be selected and arrange? in such order that the data will be seen to include the effect \& carry-over in some runs and to exclude it in others. 
methods for measurement of haemoglobin and iron (International Committee for Standardisation in Haematology, 1978a, b).

These investigations should be carried out over the whole range of clinical interest, taking cognisance of the linearity range of the instrument. The data should be calculated for all values and again separately for high, low, and mid-concentration values (see Appendix 1). To evaluate these data there should be two runs on five days of each week for a period of at least six weeks. Within the first two weeks (baseline period) 20 runs each with at least 30 samples should be performed to set up quality control samples. During the remaining four weeks there should be a further 40 runs, each with 36 quality control samples together with samples from at least 100 patients distributed among the runs, and each estimated in duplicate (1640 analyses). These same patient samples should also be measured in duplicate by an established method or a reference method if available.

Proved outliers and runs out of control are discarded for subsequent statistical analyses but included in the protocol. Calibration samples (standards) and blanks are not included in the above figures of the overall total of 2240 results, which are then used for analysis by standard statistical procedures.

A suitable protocol for use by the manufacturer in his evaluation is the NCCLS Standard PSEP-1, 1975, Protocol for establishing the precision and accuracy of automated analytic systems. * Any other protocol used by the manufacturer should first be approved by the reference laboratory (see below). When completed the protocols must be kept for at least 10 years if the instrument continues to be marketed.

\subsection{VERIFICATION OF PERFORMANCE CLAIM}

Verification of the performance claimed by the manufacturer and evaluation of the instrument as used in practice should be carried out by an authorised reference laboratory. The reference laboratory should first review the data of the original protocol provided by the manufacturer. The period of evaluation should be at least three months of regular use, with the first month primarily devoted to evaluating the manufacturer's claims and the remainder to assessing the instrument reliability in a routine ambience when used by at least five different workers (see 'Assessment of general performance' below). During this latter time the opportunity should be taken for testing the effects of specimens

*Available from National Committee for Clinical Laboratory Standards, 771 E. Lancaster Avenue, Villanova, PA 19085, USA. with high protein concentration on haemoglobin, cold agglutinins on RBC, abnormal platelets on $R B C$, etc. Each function of the instrument should be tested separately for precision and accuracy.

The following data should be obtained:

(1) At least 10 determinations on the same specimen should be performed in one day. This procedure should be repeated daily on further specimens up to a total of five days.

(2) For total precision another specimen should be analysed once a day for $\mathbf{2 0}$ working days or for a shorter period if the storage stability of the substance is less.

(3) For verification of comparability a minimum of 40 samples from patients should be used, each collected freshly in an appropriate manner and measured within two hours, in duplicate, by the instrument under evaluation and also by an established method or by a reference method when available. Specimens should be selected to span the full range of concentrations expected in clinical practice (see Appendix 1). At least half the samples must be from patients with blood disorders giving results in the abnormal (low or high) ranges.

(4) An experiment on carry-over should be performed.

The data should be evaluated by standard statistical procedures-for example, by sequential analysis or formulae which are conveniently available in the NCCLS Standard PSEP-1 and are also given by Weisbrot (1975). If the verification data differ from the manufacturer's original data the reference laboratory should extend its testing to obtain such stronger statistical significance as is necessary to ensure strict comparability to the original data provided by the manufacturer. If there are discrepancies the reference laboratory should discuss them with the manufacturer or his agent at a technical level to try to establish the reason. However, the manufacturer's representatives should not become involved with the running of the instrument during the evaluation and only that part of the evaluation carried out independently of the manufacturer may be valid for subsequent statistical analysis.

All incidents and data figures during the evaluation should be recorded in the protocol even when such data will not be used for subsequent analysis. During the course of this trial electrical safety should be checked and any potential mechanical hazards noted. Hazard of infection should also be assessed.

3.3 ASSESSMENT OF GENERAL PERFORMANCE This should be carried out concurrently with the verification programme described above. The in- 
strument should be set up in the routine laboratory during the period of evaluation. All incidents and breakdowns should be recorded as well as downtime relative to total time of operation. The machine should be used by different technicians, who should record their personal reactions to it, noting especially its design, ease of use, and potential mechanical hazards.

The instrument should also be tested by participation in an appropriate inter-laboratory proficiency assessment service. During this period of trial approximate running costs should be estimated, taking into account reagents and consumables, reference/calibration and control materials, repair and maintenance, depreciation, and staff time for operation, reagent preparation, and daily maintenance. From these the mean cost per sample can be calculated for $10,50,100$, and 500 tests per day. Information should also be obtained on the skills required, noting the level of technical training required, whether the instrument is reasonably fool-proof, its acceptability by technical staff, and the anxiety factor and onset of fatigue and/or neurosis.

At the discretion of the reference laboratory the period of testing may be extended for up to six months to allow the instrument to be used for periods by staff of other laboratories and/or for special situations to be studied-for example, use in health screening services.

The final report should contain a resumé of the evaluation with the following information specifically mentioned: (1) Range of performance facilities. (2) Does the machine meet the specification set out by the manufacturer? (3) Essential advantages (for example, efficiency, time saving) and disadvantages. (4) Suitability for different requirements and potential adaptation for other analytical procedures.

Any special critical comments arising from the evaluation should be conveyed to the manufacturer in advance of the formal adoption of the report.

3.4 LONG-TERM FOLLOW-UP OF PERFORMANCÊ Organisers of national proficiency assessmer schemes should be asked to co-operate in providin information on the performance of the instrumerf in on-going trials.

When enough instruments are in use speci\& comparability trials may be organised. Participants should be invited to send comments to the reference laboratory concerning the performance of the instrument and any other relevant information.

In cases of more complex equipment a speci, questionnaire will be sent to all known users in ordè to obtain updated information on the performances of the instruments, servicing facilities, and estimates of the life-expectancy of the instrument and its component parts (see Appendix 2).

If necessary the evaluation should be amended on the basis of this information. If indicated the evalusक्षे tion procedure (in full or selected parts, as appros priate) should be repeated or extended. Similarly, if the instrument is significantly modified by the manufacturer a further evaluation may be undere taken.

\section{References}

International Committee for Standardization in Haemå tology (1978). Recommendations for reference metho $\$$ for haemoglobinometry in human blood (ICSIF standard EP 6/2, 1977) and specifications for inters national haemiglobincyanide reference preparatio (ICSH standard EP 6/3, 1977). Journal of Clinicet Pathology, 31, February issue.

International Committee for Standardisation in Haemæa tology (1978). Recommendation for measurement of serum iron in human blood. British Journal of Haema tology, 38, In Press.

Weisbrot, I. M. (1975). Quality Control in Haematology edited by S. M. Lewis and J. F. Coster, p. 199 Academic Press, London and New York.

\section{Appendix 1}

Range of values for blood constituents occurring in clinical practice*

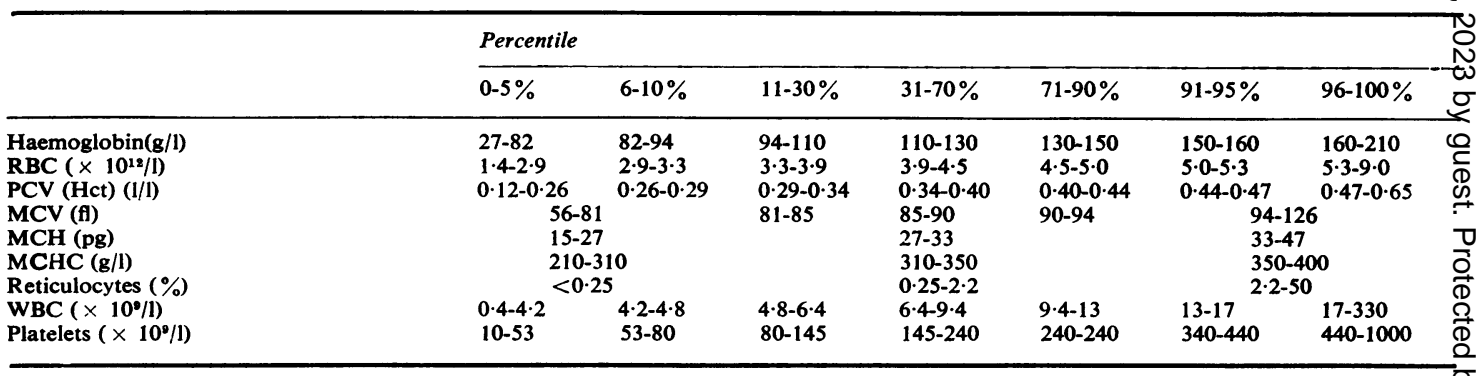

*These are approximations which are intended only as a guide to the range to be used in the testing protocol. Distribution will differ according te sex, geographic variations, and different clinical situations. 
Appendix 2-Type testing: user questionnaire

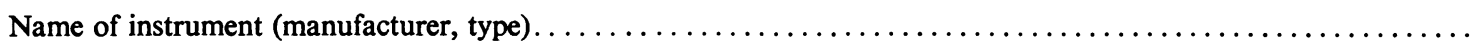

Use (tests performed; how many per week)

For what period of time have you used this instrument (in months)?

How many coworkers are acquainted with its use?

\section{CONVENIENCE}

1 Acquaintance with instrument-How easily can the average laboratory technician learn to use it?

2 Ease of maintenance-How easily can the instrument be cleaned and kept in working condition?

3 Fault finding - How easily are faults detected (warning signals)? How easily can they be located?

4 Repair-Can faults be repaired easily in situ by laboratory technician?

\section{RELIABILITY}

1 Interpretation-How direct is the relation between analysis required and method used?

2 Precision-What is the precision of the instrument?

3 Comparability-How do the results compare with those by other instruments or manual techniques?

4 Breakdowns-How many breakdowns have occurred? For how long was the instrument nonfunctioning?

3. SER VICES

1 Manual-Is there an adequate instruction manual? (language?)

2 Care-Was there a preliminary training course? What after-sale service was provided by the manufacturer or importer?

3 Fault-finding manual-Is there an easily understandable fault finding and repair service? Is it effective?

4 Servicing-How efficient is the servicing and repair department? Is there provision for loan of a stand-by instrument?

\section{COST BENEFIT}

1 Personnel-Is there saving of personnel by comparison with manual method or other instruments?

2 Running-in time-Can the equipment be installed rapidly and easily in working condition?

3 Consumption of test material-Is there increased or reduced consumption of test material and reagents compared with your previous methods?

4 Throughput time of test-How much faster is the instrument than previous methods?

\section{SUMMARY}

1 Fulfilment-Did the equipment fulfil the manufacturer's claims?

2 Value-Is the instrument worth its price in your circumstances?

3 Flexibility-Is there reasonable choice of method, reagents, and consumables for use with the instrument?

4 Conclusion-What is your overall conclusion on the instrument?

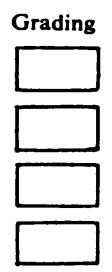

(score as 0 to 9)
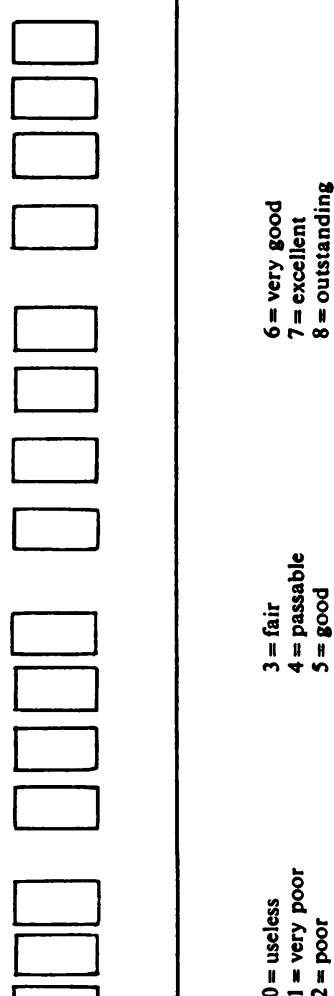